\title{
Sitagliptin Versus Ipragliflozin for Type 2 Diabetes in Clinical Practice
}

\author{
Ikuro Matsuba ${ }^{\mathrm{a}, \mathrm{d}}$, Kotaro Iemitsu ${ }^{\mathrm{a}}$, Takehiro Kawata ${ }^{\mathrm{a}}$, Takashi Iizuka ${ }^{\mathrm{a}}$, Masahiro Takihata ${ }^{\mathrm{a}}$, \\ Masahiko Takai ${ }^{a}$, Shigeru Nakajima ${ }^{\text {a }}$, Nobuaki Minamia, Shinichi Umezawa ${ }^{\text {a }}$ \\ Akira Kanamoria $^{\mathrm{a}}$, Hiroshi Takeda ${ }^{\mathrm{a}}$, Shogo Ito $^{\mathrm{a}}$, Taisuke Kikuchi ${ }^{\mathrm{a}}$, Hikaru Amemiya ${ }^{\mathrm{a}}$, \\ Mizuki Kaneshiro ${ }^{\text {a }}$, Atsuko Mokubo ${ }^{\text {a }}$, Tetsuro Takuma ${ }^{a}$, Hideo Machimura ${ }^{a}$, Keiji Tanaka ${ }^{a}$, \\ Taro Asakura ${ }^{a}$, Akira Kubota ${ }^{a}$, Sachio Aoyagi ${ }^{a}$, Kazuhiko Hoshino ${ }^{a}$, Masashi Ishikawa ${ }^{a}$, \\ Mitsuo Obana ${ }^{a}$, Nobuo Sasai ${ }^{a}$, Hideaki Kaneshige ${ }^{a}$, Masaaki Miyakawa ${ }^{a}$, \\ Yasushi Tanaka ${ }^{\mathrm{b}}$, Yasuo Terauchic
}

\begin{abstract}
Background: Dipeptidyl peptidase-4 inhibitors and sodium-glucose cotransporter-2 inhibitors are frequently used to treat type 2 diabetes. However, there have been no direct comparisons of these antidiabetic drugs in Japanese patients with type 2 diabetes.

Methods: We retrospectively assessed the effects of treatment with sitagliptin (a dipeptidyl peptidase-4 inhibitor) for 24 weeks in the ASSET-K study or treatment with ipragliflozin (a sodium-glucose cotransporter-2 inhibitor) for 24 weeks in the ASSIGN-K study. In both studies, patients with poor glycemic control received the study drug in addition to standard care with/without other antidiabetic medications or were switched to the study drug. The effects of each drug on metabolic risk factors (body weight, blood glucose, and lipids), blood pressure, and renal function were compared.
\end{abstract}

Results: After 4 weeks of treatment, hemoglobin Alc was significantly lower in patients receiving ipragliflozin than in those receiving sitagliptin, but the difference was not significant at 12 or 24 weeks. Body mass index showed a significantly larger decrease with ipragliflozin treatment than sitagliptin treatment throughout most of the study period ( $\mathrm{P}<0.001$ at 24 weeks). The mean blood pressure also showed a significantly larger decrease with ipragliflozin treatment than sitagliptin treatment throughout most of the study period ( $\mathrm{P}=$ 0.007 at 24 weeks). In contrast, the decrease of the estimated glomer-

Manuscript submitted August 27, 2019, accepted September 9, 2019

aStudy Group of the Diabetes Committee, Kanagawa Diabetes Task Force, Kanagawa Physicians Association, Kanagawa, Japan

${ }^{b}$ Division of Metabolism and Endocrinology, Department of Internal Medicine, St. Marianna University School of Medicine, Kanagawa, Japan 'Department of Endocrinology and Diabetes, Yokohama City University Medical Center, Kanagawa, Japan

${ }^{\mathrm{d} C}$ Corresponding Author: Ikuro Matsuba, Kanagawa Diabetes Task Force, Kanagawa Physicians Association, 1-3 Fujimichou, Nakaku, Yokohama, Kanagawa 231-0037, Japan. Email: ikuro@matsuba-web.com

doi: https://doi.org/10.14740/jem604 ular filtration rate after 24 weeks was significantly larger in patients treated with sitagliptin than those receiving ipragliflozin $(\mathrm{P}=0.012)$.

Conclusions: Ipragliflozin may be more effective than sitagliptin for Japanese patients with type 2 diabetes who have hypertension, obesity, and/or renal dysfunction.

Keywords: Dipeptidyl peptidase-4 inhibitor; Ipragliflozin; Sitagliptin; Sodium-glucose cotransporter-2 inhibitor; Type 2 diabetes

\section{Introduction}

In patients with type 2 diabetes mellitus (T2DM), it is important to maintain good glycemic control and to normalize both fasting and postprandial glucose levels. Various classes of antidiabetic agents with different mechanisms of actions are used to treat T2DM. Among them, dipeptidyl peptidase-4 (DPP4) inhibitors increase circulating levels of incretins, including glucagon-like peptide-1 and glucose-dependent insulinotropic polypeptide. Incretins promote insulin secretion, so DPP4 inhibitors exert a hypoglycemic effect by indirectly potentiating the secretion of insulin [1-5], making hypoglycemia unlikely in patients using these drugs. Since DPP4 inhibitors overcome some of the problems associated with older antidiabetic drugs, such as hypoglycemia and weight gain, these agents are widely used to treat T2DM. We previously performed the ASSET-K study that followed the clinical course of T2DM patients on treatment with the DPP4 inhibitor sitagliptin, revealing a decrease of hemoglobin A1c (HbAlc) after initiation of sitagliptin therapy [6], as well as a decrease of blood pressure, improvement of serum lipids [7], and various other effects [8-12].

Sodium-glucose cotransporter-2 (SGLT2) inhibitors represent another treatment option for T2DM. These drugs block the action of SGLT2 and suppress resorption of glucose in the proximal renal tubules to exert a hypoglycemic effect by promoting glucose excretion in the urine [13-15]. We also performed the ASSIGN-K study that investigated the clinical course of T2DM 
patients receiving treatment with ipragliflozin, the first SGLT2 inhibitor approved in Japan, demonstrating both a decrease of $\mathrm{HbA1c}$ and weight loss due to reduction of body fat [16-18].

If glycemic control is inadequate in patients with T2DM, another antidiabetic drug is usually added, the dose is increased, or the patient is switched to a different drug. Since DPP4 inhibitors and SGLT2 inhibitors have different mechanisms of action, it seems important to determine which of these drugs should be preferentially added to basal therapy or used for switching in order to develop more effective treatment policies for diabetes, but insufficient information is available. Min et al conducted a meta-analysis that compared DPP4 inhibitors with SGLT2 inhibitors in T2DM patients with inadequate glycemic control on insulin therapy, which revealed that adding a SGLT2 inhibitor was more effective than adding a DPP4 inhibitor for reducing $\mathrm{HbAlc}$, fasting blood glucose, and body weight, while there was no difference in the risk of hypoglycemia [19]. However, no study has directly compared the efficacy of SGLT2 inhibitors with DPP4 inhibitors as add-on therapy to basal treatment in Japanese patients with T2DM. In particular, the effect on kidney function is an important consideration when oral antidiabetic drugs are selected in Japan, where society is aging rapidly.

As diabetes progresses, the risk of renal involvement increases, including potential development of diabetic nephropathy and renal failure [20]. It has been reported that DPP4 inhibitors may affect renal function, and we previously found slight elevation of creatinine, albeit within the reference range, in patients receiving sitagliptin [21]. Chao et al investigated several DPP4 inhibitors (sitagliptin, vildagliptin, and saxagliptin) in T2DM patients, and concluded that the risk of acute kidney injury was low with all of these drugs [22]. In addition, Hattori identified a decrease of urinary albumin excretion in T2DM patients after 24 weeks of treatment with sitagliptin [23]. Conversely, it was reported that the estimated glomerular filtration rate (eGFR) was slightly lower in patients receiving sitagliptin than in those receiving placebo, indicating a decline of renal function [24]. Thus, the effect of DPP4 inhibitors on the kidneys remains unclear. SGLT2 inhibitors reduce excessive glomerular filtration and may exert a renoprotective effect by inhibiting renal inflammation and albuminuria along with reduction of blood glucose $[25,26]$, but comparison of their renal effects with those of other antidiabetic drugs has not been performed.

Accordingly, we analyzed data from the above-mentioned ASSET-K and ASSIGN-K studies to compare the effects on glycemic control and other clinical parameters when T2DM patients with inadequate glycemic control received add-on therapy or were switched to a DPP4 inhibitor (sitagliptin) or SGLT2 inhibitor (ipragliflozin).

\section{Materials and Methods}

\section{Study design}

This retrospective analysis compared sitagliptin with ipragliflozin by using anonymized data obtained during the ASSET-K and ASSIGN-K studies, which enrolled T2DM patients attend- ing outpatient clinics in Kanagawa Prefecture, Japan. In both studies, patients with poor glycemic control received the study drug in addition to standard care with/without other antidiabetic medications or were switched to the study drug. Then their clinical course was followed for 24 weeks, with data being obtained at baseline and after 4 weeks, 12 weeks, and 24 weeks. Since the present analysis was based on anonymized data from these studies, informed consent could not be obtained from the subjects. The study complied with the Declaration of Helsinki and the protocol was approved by an appropriate institutional review board before study commencement (June 17, 2014). This study was registered with the University Hospital Medical Information Network Clinical Trials Registry (No.: UMIN000014425).

\section{Subjects}

The subjects were T2DM patients who met the inclusion/exclusion criteria of the ASSET-K study (876 patients receiving add-on therapy and 437 patients switched to sitagliptin) and the ASSIGN-K study (394 patients receiving add-on therapy and 37 patients switched to ipragliflozin). The key inclusion criteria were as follows: 1 ) Age $\geq 20$ years at the time of giving informed consent; 2) Provision of written informed consent to enrollment; 3) Inadequate glycemic control by diet and exercise alone or diet, exercise, and antidiabetic drugs for $>12$ weeks; and 4) $\mathrm{HbA} 1 \mathrm{c}>6 \%$.

The key exclusion criteria were as follows: 1) A history of hypersensitivity to any component of either study drug; 2) Severe ketosis, diabetic coma, or precoma within 6 months before enrollment; 3) Severe infection, preoperative or postoperative status, or serious injury; 4) Severe renal dysfunction; 5) Other patients judged to be ineligible by the investigator; 6) Women who were pregnant, possibly pregnant, wished to become pregnant, could potentially become pregnant during the study (unless they were using appropriate contraception, had undergone sterilization, or were postmenopausal), or were breastfeeding.

\section{Assessments}

The following characteristics of the patients were investigated: sex, age, duration of diabetes, concomitant medications, body mass index (BMI), body weight, mean blood pressure, smoking, alcohol intake, HbAlc and eGFR. In addition, data were obtained on the prevalence of cerebrovascular disease, myocardial infarction/angina, arteriosclerosis obliterans, hypertension, and dyslipidemia. For evaluation of efficacy, we investigated $\mathrm{HbA1c}$, BMI, mean blood pressure, and eGFR at 4 weeks, 12 weeks, and 24 weeks after study initiation.

\section{Statistical analysis}

Changes of variables were analyzed with adjustment for age, sex, the duration of diabetes, baseline BMI, baseline mean blood pressure, baseline eGFR, and baseline $\mathrm{HbA} 1 \mathrm{c}$ by using analysis of covariance. Covariates were selected from the above-mentioned items for analysis of factors affecting the baseline value 
Table 1. Characteristics of the Patients Treated With Sitagliptin or Ipragliflozin

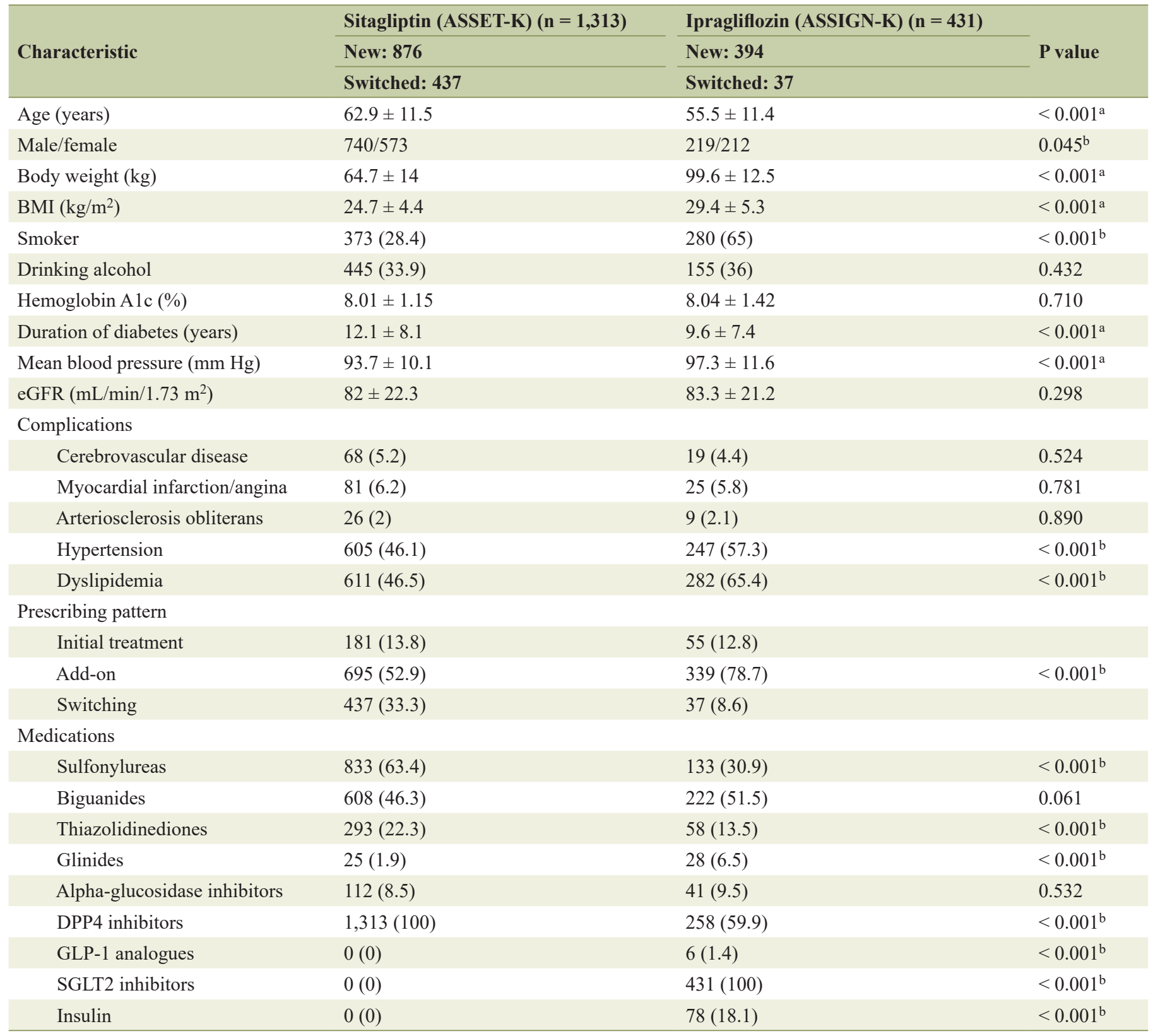

Data are the $\mathrm{n}(\%)$ or mean \pm standard deviation. ap < 0.05 Student's $t$-test; ${ }^{\text {b }}<0.05$ Chi-square test. BMI: body mass index; HbA1c: hemoglobin A1c; eGFR: estimated glomerular filtration rate; DPP4: dipeptidyl peptidase-4; GLP-1: glucagon-like peptide-1; SGLT2: sodium-glucose cotransporter-2.

of each parameter and factors affecting changes of HbA1c in both studies $[12,17,18]$. Changes from baseline at each time point of evaluation were compared by analysis of covariance, using only the patients with data for each item. We performed combined analysis of patients who received new/add-on therapy or were switched to the study drugs, and we also performed analysis limited to patients receiving new/add-on therapy.

The significance of differences in patient characteristics was assessed with Student's $t$-test or the Chi-square test, as appropriate. Continuous variables and nominal variables were reported as the mean \pm standard deviation and the number of patients (\%), respectively. Results of analysis of covariance were presented as the least squares mean \pm standard deviation. Analyses were done with $\mathrm{R}$ version 3.4.0 software (R Foundation for Statistical Computing, Vienna, Austria), and the level of significance was set at $5 \%$ (two-sided).

\section{Results}

Table 1 lists the characteristics of the patients treated with sit- 
Table 2. Changes of $\mathrm{HbA} 1 \mathrm{c}, \mathrm{BMI}$, Mean Blood Pressure, and eGFR

\begin{tabular}{|c|c|c|c|c|c|c|c|c|c|}
\hline \multirow{2}{*}{ Item } & \multicolumn{3}{|c|}{ Sitagliptin (ASSET-K) } & \multicolumn{3}{|c|}{ Ipragliflozin (ASSIGN-K) } & \multicolumn{2}{|c|}{ Difference } & \multirow{2}{*}{ P value } \\
\hline & $\mathbf{N}$ & LS mean & SE & $\mathbf{N}$ & LS mean & SE & LS mean & SE & \\
\hline \multicolumn{10}{|c|}{ eGFR (mL/min/1.73 m²) } \\
\hline$\Delta 12$ weeks & 364 & -2.84 & 0.58 & 340 & -1.97 & 0.61 & 0.87 & 0.90 & 0.333 \\
\hline$\Delta 24$ weeks & 364 & -4.15 & 0.59 & 340 & -1.88 & 0.61 & 2.28 & 0.91 & $0.012 *$ \\
\hline$\Delta 12$ weeks & 719 & -0.68 & 0.03 & 343 & -0.78 & 0.04 & 0.09 & 0.05 & 0.083 \\
\hline$\Delta 24$ weeks & 719 & -0.72 & 0.03 & 343 & -0.82 & 0.04 & 0.09 & 0.05 & 0.089 \\
\hline \multicolumn{10}{|l|}{ BMI $\left(\mathrm{kg} / \mathrm{m}^{2}\right)$} \\
\hline$\Delta 4$ weeks & 653 & 0.00 & 0.02 & 344 & -0.38 & 0.04 & 0.38 & 0.05 & $<0.001^{*}$ \\
\hline$\Delta 4$ weeks & 679 & -0.70 & 0.33 & 340 & -3.36 & 0.48 & 2.66 & 0.62 & $<0.001^{*}$ \\
\hline$\Delta 12$ weeks & 679 & -1.67 & 0.34 & 340 & -2.18 & 0.50 & 0.51 & 0.64 & 0.428 \\
\hline$\Delta 24$ weeks & 679 & -0.66 & 0.36 & 340 & -2.50 & 0.53 & 1.84 & 0.68 & $0.007 *$ \\
\hline
\end{tabular}

$P$ values were adjusted by analysis of covariance for age, sex, baseline duration of diabetes, baseline mean BMI, baseline mean blood pressure, baseline eGFR, and baseline HbA1c. HbA1c: hemoglobin A1c; BMI: body mass index; eGFR: estimated glomerular filtration rate; LS: least squares; SE: standard error. ${ }^{*} \mathrm{P}<0.05$

agliptin or ipragliflozin. There were significant differences of the following baseline parameters between the patients from the ASSET-K study receiving sitagliptin and those from the ASSIGN-K study receiving ipragliflozin: age $(\mathrm{P}<0.001)$, sex $(\mathrm{P}=0.045)$, body weight $(\mathrm{P}<0.001)$, BMI $(\mathrm{P}<0.001)$, smoking $(\mathrm{P}<0.001)$, duration of diabetes $(\mathrm{P}<0.001)$, and mean blood pressure $(\mathrm{P}<0.001)$. The baseline prevalence of hypertension and dyslipidemia also differed significantly (both $\mathrm{P}<$ 0.001). With regard to concomitant medications, significant differences were noted for the prescribing pattern and for use of sulfonylureas, thiazolidinediones, glinides, DPP4 inhibitors, glucagon like peptide 1 analogues, SGLT2 inhibitors, and insulin (all $\mathrm{P}<0.001)$.

Table 2 displays the changes of $\mathrm{HbA1c}$, BMI, mean blood pressure, and eGFR in both patient populations. When comparison was performed between sitagliptin and ipragliflozin at 4 weeks, 12 weeks, and 24 weeks after study initiation, the decrease of $\mathrm{HbA} 1 \mathrm{c}$ was significantly greater in the ASSIGN-K patients receiving ipragliflozin at 4 weeks $(-0.34 \pm 0.02 \%$ with sitagliptin vs. $-0.42 \pm 0.03 \%$ with ipragliflozin, $\mathrm{P}=0.012$ ). However, there was no significant difference between the two groups at 12 or 24 weeks. A significant difference in the change of BMI was noted after 4 weeks of treatment $\left(0.00 \pm 0.02 \mathrm{~kg} / \mathrm{m}^{2}\right.$ with sitagliptin vs. $-0.38 \pm 0.04$ $\mathrm{kg} / \mathrm{m}^{2}$ with ipragliflozin, $\left.\mathrm{P}<0.001\right)$. The decrease of BMI was significantly larger in the ASSIGN-K patients receiving ipragliflozin at 4 weeks and this difference persisted until 24 weeks. Although the change of mean blood pressure did not differ significantly between the two groups at 12 weeks, a sig- nificant difference was detected at 4 weeks $(-0.70 \pm 0.33 \mathrm{~mm}$ $\mathrm{Hg}$ with sitagliptin vs.-3.36 $\pm 0.48 \mathrm{~mm} \mathrm{Hg}$ with ipragliflozin, $\mathrm{P}<0.001)$ and also at 24 weeks $(-0.66 \pm 0.36 \mathrm{~mm} \mathrm{Hg}$ with sitagliptin vs.-2.50 $\pm 0.53 \mathrm{~mm} \mathrm{Hg}$ with ipragliflozin, $\mathrm{P}=0.007)$, with the reduction of mean blood pressure at these times being significantly larger in patients receiving ipragliflozin. The change of eGFR showed no significant difference between the two groups at 4 weeks and 12 weeks, but the decrease of eGFR was significantly larger in the ASSET-K study population with sitagliptin at 24 weeks $(-4.15 \pm 0.59 \mathrm{~mL} / \mathrm{min} / 1.73$ $\mathrm{m}^{2}$ with sitagliptin vs. $-1.88 \pm 0.61 \mathrm{~mL} / \mathrm{min} / 1.73 \mathrm{~m}^{2}$ with ipragliflozin, $\mathrm{P}=0.012$ ). When another analysis was performed that was restricted to the patients receiving add-on therapy with sitagliptin or ipragliflozin, there was no significant difference in the change of mean blood pressure between the two groups after 24 weeks. However, all of the other results were similar to those obtained by combined analysis of patients receiving add-on therapy and patients switched to each study drug (Table 3 ).

\section{Discussion}

To clarify differences between the effects of sitagliptin and ipragliflozin on glycemic control and other clinical parameters in Japanese outpatients with T2DM, we compared the changes of HbA1c, BMI, mean blood pressure, and eGFR during the previous ASSET-K study (sitagliptin) and ASSIGN-K study (ipragliflozin). We identified earlier reduction of $\mathrm{HbAlc}$, body 
Table 3. Changes of $\mathrm{HbA1c}$, BMI, Mean Blood Pressure, and eGFR (Excluding Switched Patients)

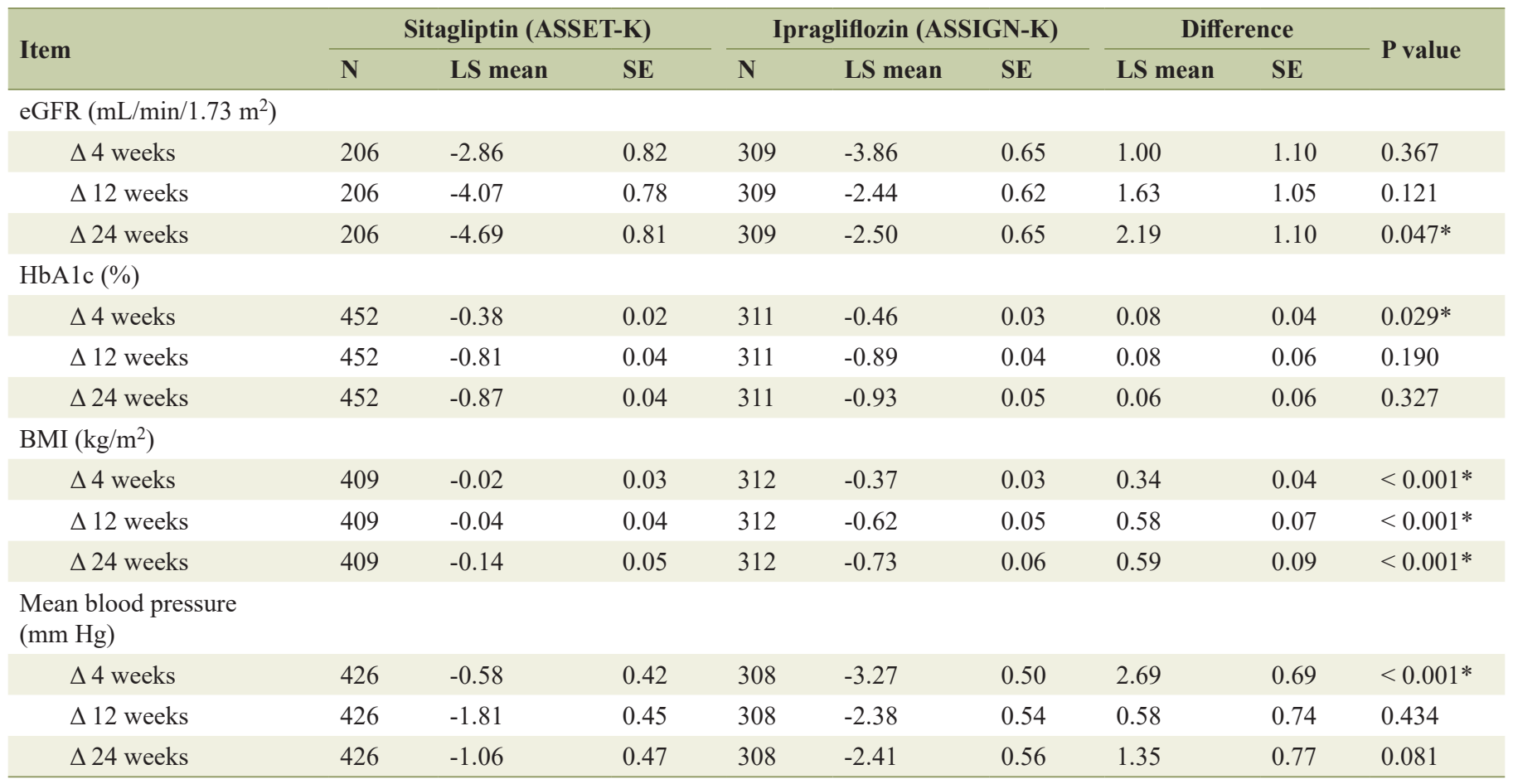

$P$ values were adjusted by analysis of covariance for age, sex, baseline duration of diabetes, baseline mean BMI, baseline mean blood pressure, baseline eGFR, and baseline HbA1c. HbA1c: hemoglobin A1c; BMI: body mass index; eGFR: estimated glomerular filtration rate; LS: least squares; SE: standard error. ${ }^{*} \mathrm{P}<0.05$

weight, and blood pressure in patients starting treatment with ipragliflozin compared to sitagliptin, suggesting that ipragliflozin achieves more rapid improvement of these parameters. In addition, eGFR was significantly lower after 24 weeks of treatment with sitagliptin than in patients receiving ipragliflozin, suggesting a renoprotective effect of the latter drug.

Significant decreases of HbA1c, BMI, and blood pressure were noted at 4 weeks after initiation of ipragliflozin treatment in the ASSIGN-K study and these improvements persisted until 24 weeks. Although eGFR also showed a significant decrease at 4 weeks after starting ipragliflozin therapy, the decline was smaller at 12 and 24 weeks [18]. In the ASSET-K study, we found a significant decrease of $\mathrm{HbAlc}$, blood pressure, and eGFR after 4 weeks of sitagliptin treatment, with persistence of these changes until 24 weeks, but no change of body weight $[7,12]$. Comparing the responses to sitagliptin and ipragliflozin, the change of HbAlc was significantly smaller at 4 weeks after initiation of ipragliflozin therapy. However, there was no difference of glycemic control between sitagliptin and ipragliflozin after 12 weeks. On the other hand, the decrease of BMI was significantly larger in the ASSIGN-K patients receiving ipragliflozin than in the ASSET-K patients receiving sitagliptin at all times of assessment, indicating that weight loss was greater in patients receiving new/add-on therapy with ipragliflozin or switching to it. The decrease of BMI with ipragliflozin was probably related to an increase of urinary glucose excretion [13-15, 25, 26], since this would reduce the net calorie intake and promote weight loss. Accordingly, ipragliflozin may be a more effective treatment option for achieving weight loss in obese T2DM patients, while sitagliptin may be preferable for lean patients.

When the change of mean blood pressure was compared between the two patient populations, there was no significant difference from baseline to 12 weeks of administration. However, the decrease of mean blood pressure was significantly larger in the patients on ipragliflozin after 4 and 24 weeks. While the underlying mechanisms have not been completely clarified, it was reported that SGLT2 inhibitors may reduce blood pressure through weight loss, improvement of arteriosclerosis, and osmotic diuresis [15-17]. This study identified a greater decrease of BMI in patients receiving ipragliflozin, which may have contributed to their lower mean blood pressure. We previously reported that blood pressure was significantly reduced after 12 weeks of sitagliptin treatment [7]. On the other hand, blood pressure showed a greater decrease during ipragliflozin therapy in the present study, suggesting that it may be more useful than sitagliptin for T2DM patients with hypertension.

The change of eGFR from baseline to 4 weeks of administration was smaller in patients receiving sitagliptin, but the difference between the two groups was reversed at 12 weeks, and the decrease of eGFR was significantly larger with sitagliptin than ipragliflozin after 24 weeks. Ipragliflozin may reduce the burden on the kidneys by suppressing glucose excretion, resulting in the smaller decrease of eGFR after 24 weeks. It has been reported that reduction of BMI and blood pressure can 
affect kidney function [18]. In this study, baseline BMI and mean blood pressure were lower in the ASSET-K patients receiving sitagliptin than in the ASSIGN-K patients receiving ipragliflozin, but the decrease of eGFR was still significantly larger during sitagliptin treatment. More than $80 \%$ of a dose of sitagliptin is excreted via the kidneys as the unchanged drug $[27,28]$ versus approximately $1 \%$ of a dose of ipragliflozin [29], suggesting that sitagliptin may impose a greater burden on the kidneys. In the present study, the change of eGFR after 4 weeks was larger with ipragliflozin than with sitagliptin. Similarly, it was reported that eGFR decreased after 6 weeks in T2DM patients receiving another SGLT2 inhibitor (canagliflozin), but returned to near baseline after 26 weeks [30]. The greater decrease of eGFR up to 4 weeks after initiation of ipragliflozin compared with sitagliptin may be explained by changes of tubuloglomerular feedback. When glucose reabsorption is suppressed by SGLT2 inhibitors, the sodium concentration increases in the proximal renal tubules and tubuloglomerular feedback is activated to reduce ultrafiltration, as has been demonstrated in an animal study and in patients with type 1 diabetes [31,32]. Although eGFR was decreased after 4 weeks of ipragliflozin treatment, it recovered subsequently, while eGFR continued to decline as the duration of sitagliptin administration became longer. These results suggest that the long-term effect of ipragliflozin on kidney function could be more beneficial than that of sitagliptin.

Several classes of oral antidiabetic drugs are available for treating type 2 diabetes, and combined therapy with SGLT2 inhibitors and DPP4 inhibitors has attracted attention. When a SGLT2 inhibitor or placebo was added to basal therapy in T2DM patients receiving metformin and DPP4 inhibitors, the SGLT2 inhibitor group showed a greater decrease of body weight, fasting plasma glucose, and $\mathrm{HbA1c}$, as well as a higher rate of achieving $\mathrm{HbA} 1 \mathrm{c}<7 \%[33,34]$. It has been reported that ipragliflozin does not influence the pharmacokinetics of sitagliptin, pioglitazone, or glimepiride, and vice versa [35], suggesting that adding ipragliflozin to such antidiabetic drugs is a reasonable option.

This study had several limitations. First, it was a retrospective analysis of combined data from two previous studies, so randomization could not be performed. Second, although we reduced bias as much as possible by performing adjusted analysis, there were still differences of patient characteristics between the two study populations and we could not exclude a possible influence on the results. Third, we could not compare kidney function based on urinary albumin excretion because albumin data were only obtained in one study. Accordingly, a long-term prospective comparison between sitagliptin and ipragliflozin would be required to confirm our findings obtained up to 24 weeks of treatment.

\section{Conclusions}

The present comparison of sitagliptin and ipragliflozin in Japanese T2DM patients with poor baseline glycemic control revealed no difference in the reduction of HbAlc after 24 weeks of treatment with either drug. However, the improvement of BMI and mean blood pressure was significantly larger in pa- tients receiving ipragliflozin than in those receiving sitagliptin. Accordingly, ipragliflozin may be a superior option for T2DM patients with hypertension or obesity. In addition, the decrease of eGFR was significantly larger in patients receiving sitagliptin than in those receiving ipragliflozin, suggesting that ipragliflozin may more effectively reduce the burden on the kidneys.

\section{Acknowledgments}

ASSIGN-K is a prospective study that is currently in progress under the Kanagawa Physicians Association. We would like to thank the physicians and staff of the Kanagawa Physicians Association involved in this research and the patients participating in this study.

\section{Financial Declaration}

This work was supported by Astellas Pharma, Inc. The company was not involved in the study design, patient enrollment, data aggregation and analysis, data interpretation or preparation of this report.

\section{Conflict of Interest}

The authors have no conflict of interest to report.

\section{Informed Consent}

Not applicable.

\section{Author Contributions}

IM and TK were involved in the acquisition and analysis of data and wrote the first draft of the manuscript. IM designed the study. IM and TK were involved in the statistical analysis. IM, Y. Tanaka, and Y. Terauchi performed the model analysis. All authors reviewed and edited the manuscript critically for important intellectual content. IM is the guarantor of this work and, as such, had full access to all the data in the study and takes responsibility for the integrity of the data and the accuracy of the data analysis. All authors agree to be accountable for all aspects of the work in ensuring that questions related to the accuracy or integrity of any part of the work are appropriately investigated and resolved.

\section{References}

1. Herman GA, Stein PP, Thornberry NA, Wagner JA. Dipeptidyl peptidase-4 inhibitors for the treatment of type 2 diabetes: focus on sitagliptin. Clin Pharmacol Ther. 2007;81(5):761-767.

2. Herman GA, Stevens C, Van Dyck K, Bergman A, Yi B, 
De Smet M, Snyder K, et al. Pharmacokinetics and pharmacodynamics of sitagliptin, an inhibitor of dipeptidyl peptidase IV, in healthy subjects: results from two randomized, double-blind, placebo-controlled studies with single oral doses. Clin Pharmacol Ther. 2005;78(6):675688.

3. Bergman AJ, Stevens C, Zhou Y, Yi B, Laethem M, De Smet M, Snyder K, et al. Pharmacokinetic and pharmacodynamic properties of multiple oral doses of sitagliptin, a dipeptidyl peptidase-IV inhibitor: a double-blind, randomized, placebo-controlled study in healthy male volunteers. Clin Ther. 2006;28(1):55-72.

4. Herman GA, Bergman A, Stevens C, Kotey P, Yi B, Zhao P, Dietrich B, et al. Effect of single oral doses of sitagliptin, a dipeptidyl peptidase-4 inhibitor, on incretin and plasma glucose levels after an oral glucose tolerance test in patients with type 2 diabetes. J Clin Endocrinol Metab. 2006;91(11):4612-4619.

5. Seino Y, Fukushima M, Yabe D. GIP and GLP-1, the two incretin hormones: Similarities and differences. J Diabetes Investig. 2010;1(1-2):8-23.

6. Maeda H, Kubota A, Tanaka Y, Terauchi Y, Matsuba I, ASSET-K Study group. The safety, efficacy and predictors for HbA1c reduction of sitagliptin in the treatment of Japanese type 2 diabetes. Diabetes Res Clin Pract. 2012;95(1):e20-22.

7. Kubota A, Maeda H, Kanamori A, Matoba K, Jin Y, Minagawa F, Obana $\mathrm{M}$, et al. Pleiotropic effects of sitagliptin in the treatment of type 2 diabetes mellitus patients. J Clin Med Res. 2012;4(5):309-313.

8. Kubota A, Maeda H, Kanamori A, Matoba K, Jin Y, Minagawa F, Obana M, et al. Efficacy and safety of sitagliptin monotherapy and combination therapy in Japanese type 2 diabetes patients. J Diabetes Investig. 2012;3(6):503-509.

9. Kanamori A, Matsuba I. Factors associated with reduced efficacy of sitagliptin therapy: analysis of 93 patients with type 2 diabetes treated for 1.5 years or longer. J Clin Med Res. 2013;5(3):217-221.

10. Maeda H, Kubota A, Kanamori A, Tanaka Y, Terauchi Y, Matsuba I, ASSET-K Study group. Long-term efficacy and safety of sitagliptin in the treatment of Japanese Type 2 diabetes (ASSET-K1) to a target of HbA1c $<7 \%$. J Endocrinol Invest. 2013;36(8):568-573.

11. Umezawa S, Kubota A, Maeda H, Kanamori A, Matoba $\mathrm{K}$, Jin Y, Minagawa F, et al. Two-year assessment of the efficacy and safety of sitagliptin in elderly patients with type 2 diabetes: Post hoc analysis of the ASSET-K study. BMC Endocr Disord. 2015;15:34.

12. Yuasa S, Sato K, Takai M, Ishikawa M, Umezawa S, Kubota A, Maeda H, et al. Factor analysis of changes in hemoglobin A1c after 12 months of sitagliptin therapy in patients with type 2 diabetes. J Clin Med Res. 2016;8(6):461-471.

13. Bailey CJ, Gross JL, Pieters A, Bastien A, List JF. Effect of dapagliflozin in patients with type 2 diabetes who have inadequate glycaemic control with metformin: a randomised, double-blind, placebo-controlled trial. Lancet. 2010;375(9733):2223-2233.

14. Komoroski B, Vachharajani N, Feng Y, Li L, Kornhauser
D, Pfister M. Dapagliflozin, a novel, selective SGLT2 inhibitor, improved glycemic control over 2 weeks in patients with type 2 diabetes mellitus. Clin Pharmacol Ther. 2009;85(5):513-519.

15. Komoroski B, Vachharajani N, Boulton D, Kornhauser D, Geraldes M, Li L, Pfister M. Dapagliflozin, a novel SGLT2 inhibitor, induces dose-dependent glucosuria in healthy subjects. Clin Pharmacol Ther. 2009;85(5):520526.

16. Iizuka T, Iemitsu K, Takihata M, Takai M, Nakajima S, Minami N, Umezawa S, et al. Efficacy and safety of ipragliflozin in Japanese patients with type 2 diabetes: Interim Outcome of the ASSIGN-K Study. J Clin Med Res. 2016;8(2):116-125.

17. Iemitsu K, Iizuka T, Takihata M, Takai M, Nakajima S, Minami N, Umezawa S, et al. Factors influencing changes in hemoglobin A1c and body weight during treatment of type 2 diabetes with ipragliflozin: interim analysis of the ASSIGN-K study. J Clin Med Res. 2016;8(5):373378.

18. Iemitsu K, Kawata T, Iizuka T, Takihata M, Takai M, Nakajima S, Minami N, et al. Effectiveness of ipragliflozin for reducing hemoglobin a1c in patients with a shorter type 2 diabetes duration: interim report of the ASSIGN-K study. J Clin Med Res. 2017;9(9):793-801.

19. Min SH, Yoon JH, Hahn S, Cho YM. Comparison between SGLT2 inhibitors and DPP4 inhibitors added to insulin therapy in type 2 diabetes: a systematic review with indirect comparison meta-analysis. Diabetes Metab Res Rev. 2017;33(1):e2818.

20. Rao Kondapally Seshasai S, Kaptoge S, Thompson A, Di Angelantonio E, Gao P, Sarwar N, Whincup PH, et al. Diabetes mellitus, fasting glucose, and risk of cause-specific death. N Engl J Med. 2011;364(9):829-841.

21. Maeda H, Kubota A, Kanamori A, Tanaka Y, Terauchi Y, Matsuba I, Study Group of Diabetes Committee KPA. Effects of sitagliptin on the serum creatinine in Japanese type 2 diabetes. Diabetes Res Clin Pract. 2015;108(3):e42-45.

22. Chao CT, Wang J, Wu HY, Chien KL, Hung KY. Dipeptidyl peptidase 4 inhibitor use is associated with a lower risk of incident acute kidney injury in patients with diabetes. Oncotarget. 2017;8(32):53028-53040.

23. Hattori S. Sitagliptin reduces albuminuria in patients with type 2 diabetes. Endocr J. 2011;58(1):69-73.

24. Cornel JH, Bakris GL, Stevens SR, Alvarsson M, Bax WA, Chuang LM, Engel SS, et al. Effect of sitagliptin on kidney function and respective cardiovascular outcomes in type 2 diabetes: outcomes from TECOS. Diabetes Care. 2016;39(12):2304-2310.

25. Kalra S, Singh V, Nagrale D. Sodium-Glucose Cotransporter-2 Inhibition and the Glomerulus: A Review. Adv Ther. 2016;33(9):1502-1518.

26. Novikov A, Vallon V. Sodium glucose cotransporter 2 inhibition in the diabetic kidney: an update. Curr Opin Nephrol Hypertens. 2016;25(1):50-58.

27. Bergman AJ, Cote J, Yi B, Marbury T, Swan SK, Smith W, Gottesdiener K, et al. Effect of renal insufficiency on the pharmacokinetics of sitagliptin, a dipeptidyl peptidase-4 inhibitor. Diabetes Care. 2007;30(7):1862-1864. 
28. Eligar VS, Bain SC. A review of sitagliptin with special emphasis on its use in moderate to severe renal impairment. Drug Des Devel Ther. 2013;7:893-903.

29. Kadokura T, Saito M, Utsuno A, Kazuta K, Yoshida S, Kawasaki S, Nagase I, et al. Ipragliflozin (ASP1941), a selective sodium-dependent glucose cotransporter 2 inhibitor, safely stimulates urinary glucose excretion without inducing hypoglycemia in healthy Japanese subjects. Diabetol Int. 2011;2:172-182.

30. Yamout H, Perkovic V, Davies M, Woo V, de Zeeuw D, Mayer C, Vijapurkar U, et al. Efficacy and safety of canagliflozin in patients with type 2 diabetes and stage 3 nephropathy. Am J Nephrol. 2014;40(1):64-74.

31. Cherney DZ, Perkins BA, Soleymanlou N, Maione M, Lai V, Lee A, Fagan NM, et al. Renal hemodynamic effect of sodium-glucose cotransporter 2 inhibition in patients with type 1 diabetes mellitus. Circulation. 2014;129(5):587597.

32. Thomson SC, Rieg T, Miracle C, Mansoury H, Whaley
J, Vallon V, Singh P. Acute and chronic effects of SGLT2 blockade on glomerular and tubular function in the early diabetic rat. Am J Physiol Regul Integr Comp Physiol. 2012;302(1):R75-83.

33. Mathieu C, Ranetti AE, Li D, Ekholm E, Cook W, Hirshberg B, Chen H, et al. Randomized, double-blind, phase 3 trial of triple therapy with dapagliflozin add-on to Saxagliptin plus metformin in type 2 diabetes. Diabetes Care. 2015;38(11):2009-2017.

34. Rodbard HW, Seufert J, Aggarwal N, Cao A, Fung A, Pfeifer M, Alba M. Efficacy and safety of titrated canagliflozin in patients with type 2 diabetes mellitus inadequately controlled on metformin and sitagliptin. Diabetes Obes Metab. 2016;18(8):812-819.

35. Smulders RA, Zhang W, Veltkamp SA, van Dijk J, Krauwinkel WJ, Keirns J, Kadokura T. No pharmacokinetic interaction between ipragliflozin and sitagliptin, pioglitazone, or glimepiride in healthy subjects. Diabetes Obes Metab. 2012;14(10):937-943. 